Physics Basis for the Fusion Ignition Research Experiment (FIRE)

by

D.M. Meade, R.J. Thome, N.R. Sauthoff, P.J. Heitzenroeder, B.E. Nelson, M.A. Ulrickson, C.E. Kessel, J.H. Schultz, P.H. Rutherford, J.C. Wesley, K.M. Young, W.M. Nevins, N.A. Uckan, and J.A. Schmidt

July 2000

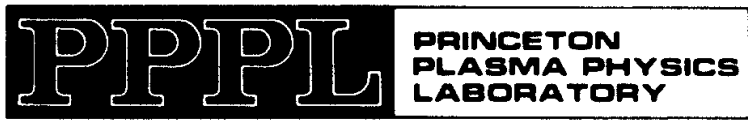

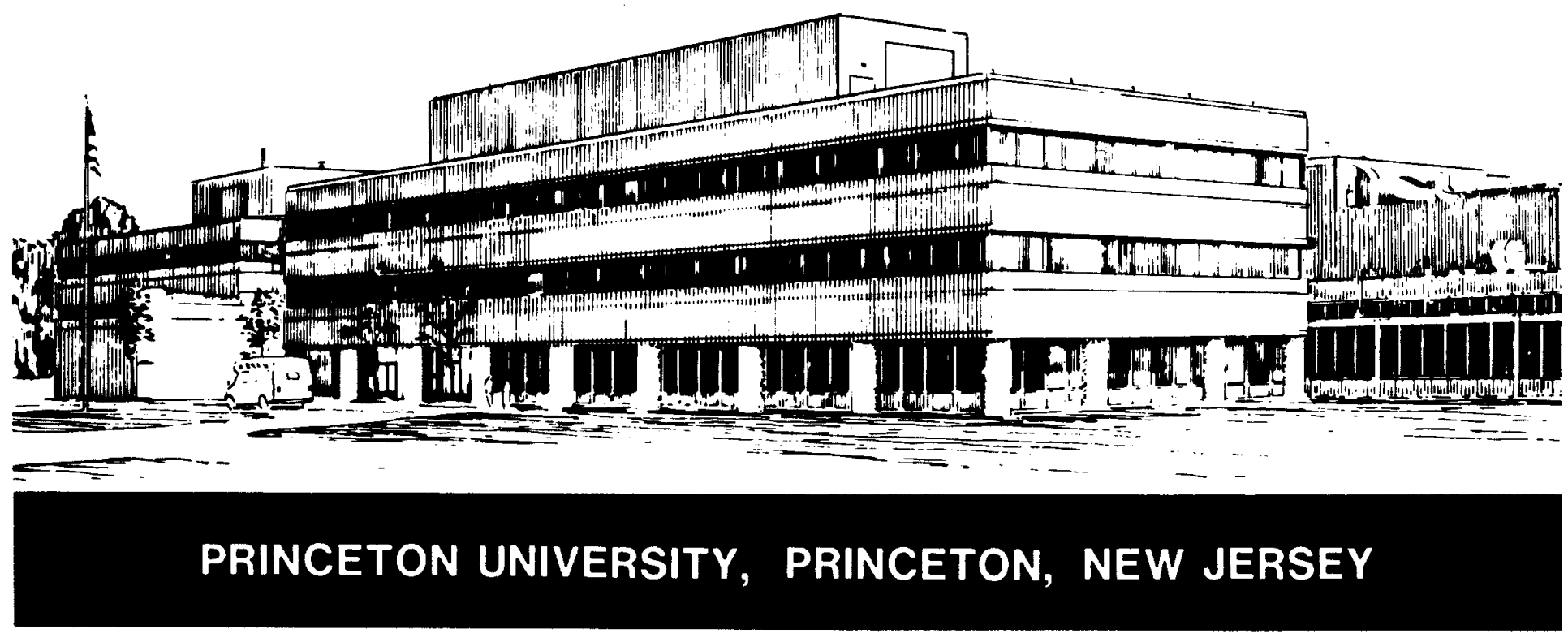




\section{PPPL Reports Disclaimer}

This report was prepared as an account of work sponsored by an agency of the United States Government. Neither the United States Government nor any agency thereof, nor any of their employees, makes any warranty, express or implied, or assumes any legal liability or responsibility for the accuracy, completeness, or usefulness of any information, apparatus, product, or process disclosed, or represents that its use would not infringe privately owned rights. Reference herein to any specific commercial product, process, or service by trade name, trademark, manufacturer, or otherwise, does not necessarily constitute or imply its endorsement, recommendation, or favoring by the United States Government or any agency thereof. The views and opinions of authors expressed herein do not necessarily state or reflect those of the United States Government or any agency thereof.

\section{Availability}

This report is posted on the U.S. Department of Energy's Princeton Plasma Physics Laboratory Publications and Reports web site in Calendar Year 2000. The home page for PPPL Reports and Publications is: http://www.pppl.gov/pub_report/

DOE and DOE Contractors can obtain copies of this report from:

U.S. Department of Energy

Office of Scientific and Technical Information

DOE Technical Information Services (DTIS)

P.O. Box 62

Oak Ridge, TN 37831

Telephone: (865) 576-8401

Fax: (865) 576-5728

Email: reports@adonis.osti.gov

This report is available to the general public from:

National Technical Information Service

U.S. Department of Commerce

5285 Port Royal Road

Springfield, VA 22161

Telephone: $1-800-553-6847$ or

(703) $605-6000$

Fax: (703) 321-8547

Internet: http://www.ntis.gov/ordering.htm 


\title{
Physics Basis for the Fusion Ignition Research Experiment (FIRE)
}

\author{
D. M. Meade ${ }^{1}$, R. J. Thome ${ }^{2}$, N. R. Sauthoff ${ }^{1}$, P. J. Heitzenroeder ${ }^{1}$, B. E. \\ Nelson $^{3}$, M.A Ulrickson ${ }^{4}$, C. E. Kessel ${ }^{1}$, J. H. Schultz ${ }^{2}$, P. H. Rutherford ${ }^{1}$, J. C. \\ Wesley $^{5}$, K. M. Young ${ }^{1}$, W. M. Nevins ${ }^{6}$, N. A. Uckan ${ }^{3}$ and \\ J. A. Schmidt ${ }^{1}$ \\ ${ }^{I}$ Princeton Plasma Physics Laboratory, Princeton, NJ 08543, USA \\ ${ }^{2}$ Massachusetts Institute of Technology, Cambridge, MA 02139, USA \\ ${ }^{3}$ Oak Ridge National Laboratory, Oak Ridge, TN 37831, USA \\ ${ }^{4}$ Sandia National Laboratory, Albuquerque, NM 87185, USA \\ ${ }^{5}$ General Atomics, San Diego, CA 92186, USA \\ ${ }^{6}$ Lawrence Livermore National Laboratory, Livermore, CA 94551, USA
}

Understanding the properties of high gain (alpha-dominated) fusion plasmas in an advanced toroidal configuration is a critical issue that must be addressed to provide the scientific foundation for an attractive magnetic fusion reactor. The functional fusion plasma objectives for major next physics steps in magnetic fusion research can be described as:

Burning Plasma Physics - The achievement and understanding of alphadominated plasmas that have characteristics similar to those expected in a fusion energy source, and

Advanced Toroidal Physics - The achievement and understanding of bootstrap-current-dominated plasmas with externally controlled profiles and other characteristics (e.g., confinement and $\beta$ ) similar to those expected in an attractive fusion system.

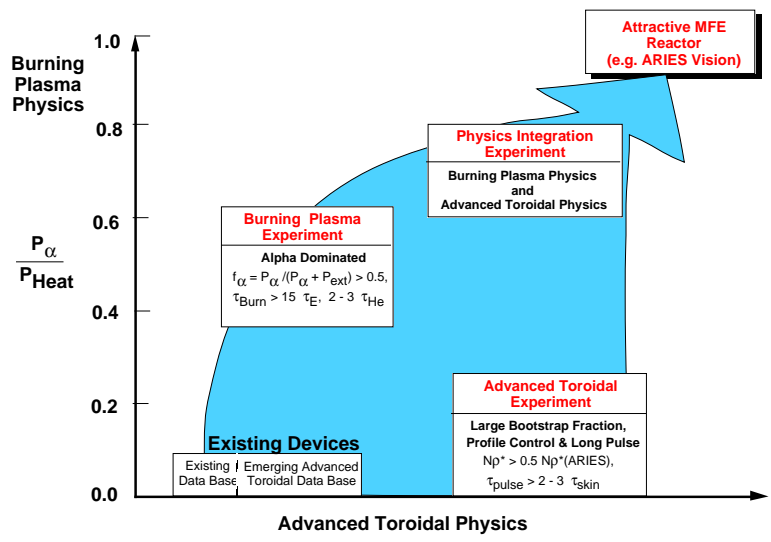

Fig.1 Next Physics Steps in Magnetic Fusion

These requirements lead naturally to a set of fusion physics Stepping Stones as illustrated in Fig. 1. The ranges of plasma performance and duration to address these issues are shown schematically with the natural time scales for important plasma processes.

A design study of a Fusion Ignition Research Experiment (FIRE) is underway to investigate near term opportunities for advancing the scientific understanding of self-heated fusion plasmas in advanced toroidal configurations. The emphasis is on understanding the behavior of plasmas dominated by alpha heating $(\mathrm{Q}>5)$ that are sustained sufficiently long compared to most characteristic plasma time scales $\left(\sim 30 \tau_{\mathrm{E}}, \sim 6 \tau_{\mathrm{He}}, \sim \tau_{\text {skin }}\right.$, where $\tau_{\mathrm{He}}$ is the helium ash confinement time at $5 \tau_{\mathrm{E}}$, and $\tau_{\text {skin }}$ is the time for the plasma current profile to redistribute at fixed total current) to allow the evolution of alpha defined profiles. The programmatic mission of FIRE is to attain, explore, understand and optimize alpha-dominated plasmas to provide knowledge for the design of attractive magnetic fusion energy systems. The programmatic strategy is to access the alpha-dominated regimes with confidence using the 
present tokamak data base (e.g., Elmy-H-mode, $\leq 0.75$ Greenwald density) while maintaining the flexibility for accessing and exploring advanced tokamak modes at lower magnetic fields and fusion power for longer durations in later stages of the experimental program. A major goal is to develop a design concept that would meet these physics objectives with a tokamak (load assembly) construction cost of $\sim \$ 300 \mathrm{M}$ and a total project cost in the range of $\$ 1 \mathrm{~B}$.

The activities have focused on the technical evaluation of a compact, high-field, highlyshaped tokamak with the parameters shown in Table I. The philosophy of FIRE is to challenge, and extend existing physics limits toward the regimes envisioned for a fusion reactor. Confinement projections are uncertain, and one of the major objectives of a next step

Table I. Design Goals for FIRE

\begin{tabular}{|l|c|}
\hline $\mathrm{R}(\mathrm{m}), \mathrm{a}(\mathrm{m})$ & $2.0,0.525$ \\
\hline$\kappa_{95}, \delta_{95}$ & $\approx 1.8, \approx 0.4$ \\
\hline $\mathrm{q}_{95}$ & $>3$ \\
\hline $\mathrm{B}_{\mathrm{t}}\left(\mathrm{R}_{\mathrm{o}}\right)(\mathrm{T})$ & $10(12)^{*}$ \\
\hline Wmag TF $(\mathrm{GJ})$ & 3.7 \\
\hline $\mathrm{I}_{\mathrm{p}}(\mathrm{MA})$ & $6.44(7.7)^{*}$ \\
\hline flattop time $(\mathrm{s})$ & $\sim 20(12)^{*}$ \\
\hline alpha heating fraction & $>0.5$ \\
\hline$\tau_{\mathrm{E}}, \tau_{\text {skin }}(\mathrm{s})$ & $\sim 0.6, \sim 13$ \\
\hline $\mathrm{Z}_{\text {eff }}\left(3 \%\right.$ Be + He $\left.\left(5 \tau_{\mathrm{E}}\right)\right)$ & 1.4 \\
\hline Fusion Power $(\mathrm{MW})$ & $\sim 200$ \\
\hline ICRF Power $(\mathrm{MW})$ & 30 \\
\hline Tokamak Cost $(\$ B)$ & $\sim 0.3$ \\
\hline Project Cost $(\$ B)$ & $\sim 1$ \\
\hline
\end{tabular}

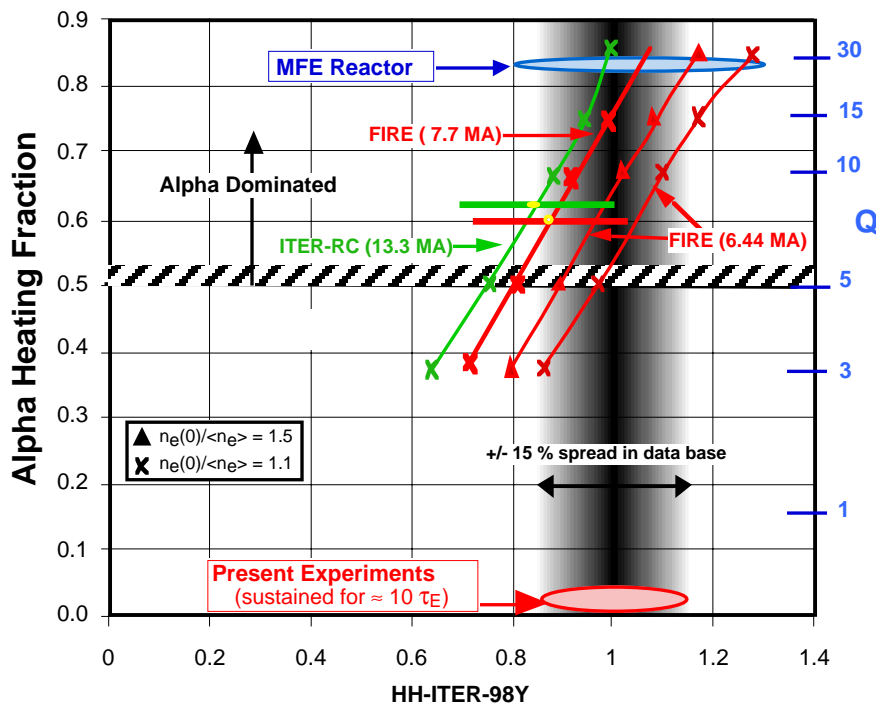

* Upgrade capability

Fig.2 H-Mode Projections using ITER IPB98(y)

experiment is to extend the experimental range beyond existing experiments and capability to test projections closer to reactor conditions. The plasma performance of FIRE was estimated using a zero-dimension analysis with energy confinement given by ITER IPB98(y) [1] for the Elmy H-mode, $\beta_{\mathrm{N}} \leq 2.5$, density $\leq 0.75$ Greenwald density, $\mathrm{P}_{\text {threshold }} \geq\left(0.9 / \mathrm{A}_{\mathrm{i}}\right) \mathrm{n}^{0.75} \mathrm{BR}^{2}, 3 \%$ Be impurities and alpha ash accumulating self consistently with $\tau_{\mathrm{He}}=5 \tau_{\mathrm{E}}$. The results are shown in Fig. 2 for two values of density profile peaking. For the baseline FIRE parameters $(6.44 \mathrm{MA} / 10 \mathrm{~T})$, the alpha heating fraction, $\mathrm{f}_{\alpha}$, rises from $\approx 40 \%$ at the low end of the present database to $\approx 80 \%$ at the high end. Note that the alpha heating fraction, which is important for physics studies, is more robust to confinement uncertainty and rises linearly with confinement while Q changes rapidly as ignition is approached. These parameters extend the capability to study alpha heating effects sustained for $>10 \tau_{\mathrm{E}}$ more than an order of magnitude beyond previous experiments. Additional performance (Table II.) could be obtained by adding additional power supplies and operating at $7.7 \mathrm{MA}(12 \mathrm{~T})$ with a reduction in toroidal field flattop to $12 \mathrm{~s}$ thereby providing additional physics margin. The H-mode power threshold poses a challenge for high field tokamaks and the power transported across the separatrix in FIRE marginally exceeds the projected L-H transition threshold.

\begin{tabular}{|l|c|c|c|c|c|c|c|c|}
\hline Table II. & $\mathrm{B}_{\mathrm{T}}(\mathrm{T})$ & $\mathrm{I}_{\mathrm{p}}(\mathrm{MA})$ & $\mathrm{f}_{\alpha}$ & $\mathrm{Q}$ & $\mathrm{n} / \mathrm{n}_{\mathrm{GW}}$ & $\mathrm{HH} 98(\mathrm{y})$ & $\beta_{\mathrm{N}}$ & $\mathrm{P}_{\text {trans }} / \mathrm{P}_{\text {thres }}$ \\
\hline Baseline & 10 & 6.44 & 0.66 & 10 & 0.7 & 1.04 & 2.5 & 1.15 \\
\hline Upgrade & 12 & 7.7 & 0.66 & 10 & 0.6 & 0.85 & 1.9 & 1.09 \\
\hline
\end{tabular}


A Tokamak Simulation Code (TSC) simulation indicates that alpha-dominated plasmas can be sustained for $>30 \tau_{\mathrm{E}},>5 \tau_{\mathrm{He}}$ and $~$ $1 \tau_{\text {skin }}$ as shown in Fig. 3. This example shows the importance of having sufficient magnetic field flattop for plasma startup ( 4 s), helium ash evolution ( $\sim \mathrm{s})$ prior to achieving a steady burn for experimental studies. In addition, capability must be provided for controlled plasma shutdown without causing a disruption on every pulse. The primary methods

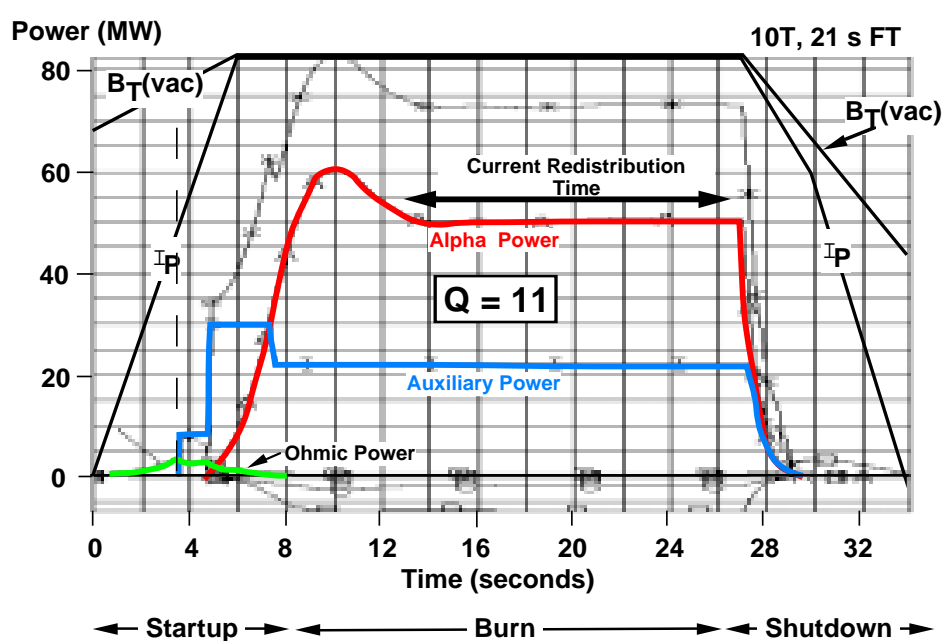

Fig. 3. Evolution of an alpha-dominated discharge. of burn control will be to adjust the input power and the D-T fueling rate.

A longer term goal of FIRE is to explore advanced tokamak regimes using pellet injection and current ramps to create reversed shear plasmas (e.g., PEP modes) for durations of 1 to 3 current redistribution times. This AT capability is expected to produce modestly enhanced confinement and beta as observed in present large tokamak experiments, and provides a continuous transition from $\mathrm{H}$-mode operation to advanced tokamak operation. A range of advanced tokamak modes could then be studied on FIRE. If $\mathrm{HH} 98(\mathrm{y}, 1) \approx 1.2$ and $\beta_{\mathrm{N}} \approx 3$ can be attained and sustained at $6.5 \mathrm{MA}$, then inductively-driven plasmas with $\mathrm{Q}>>10$ could be achieved at full field for durations of $\sim 20 \mathrm{~s}\left(\sim 1 \tau_{\text {skin }}\right)$. The cryogenically cooled coil systems on FIRE are capable of long pulses at reduced fields: $20 \mathrm{~s}$ at $10 \mathrm{~T} ; 40 \mathrm{~s}$ at $8 \mathrm{~T}$ and $90 \mathrm{~s}$ at $6 \mathrm{~T}$. Therefore, the magnetic field and plasma current for the modestly advanced performance case can be reduced by $20 \%$ resulting in pulses $\sim 40 \mathrm{~s}\left(\sim 3 \tau_{\text {skin }}\right)$ long that have $\mathrm{Q}>5$. Selfconsistent physics scenarios and engineering solutions for power handling are not yet developed for the longer pulse ( $40 \mathrm{~s})$ scenarios.

The baseline magnetic fields and pulse lengths can be provided with $\mathrm{BeCu} / \mathrm{OFHC}$ toroidal field (TF) coils and OFHC poloidal field (PF) coils that are pre-cooled to $77^{\circ} \mathrm{K}$ prior to the pulse and allowed to warm up to $373{ }^{\circ} \mathrm{K}$ at the end of the pulse. The crosssection of FIRE is shown schematically in Figure 4. The key "advanced tokamak" features are: strong plasma shaping, double null poloidal divertors, low TF ripple ( 0.34\%@ outer midplane), internal control coils and space for yet to be determined wall stabilization capabilities. The 16 TF coil system is wedged with a compression ring to

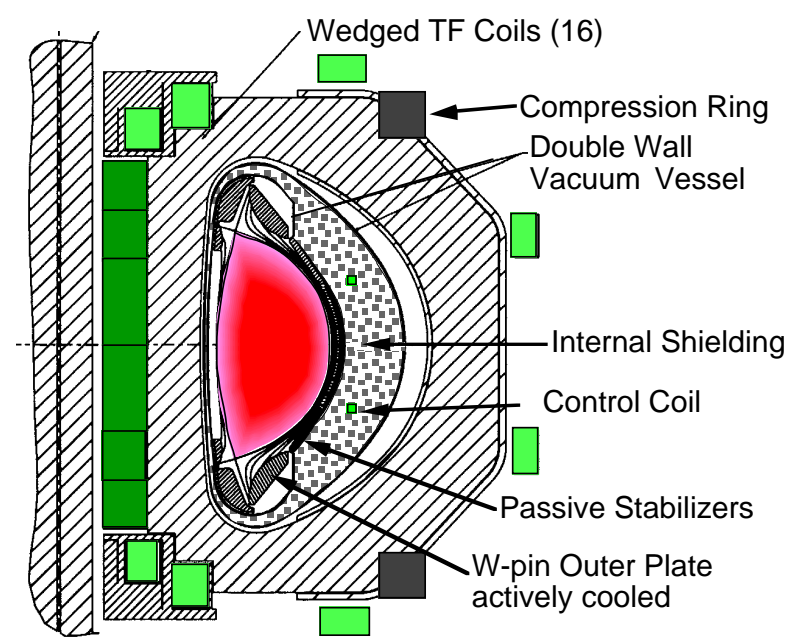

Fig. 4. Cross-section of FIRE resist de-wedging at the top and bottom of the inner TF leg. Shielding is added between the walls of a double wall vacuum vessel to reduce nuclear heating of the coils, limit insulation dose and allow hands-on maintenance outside the envelope of the TF coils within a few hours after a full power D-T shot. Large $(1.3 \mathrm{~m}$ by $0.7 \mathrm{~m})$ midplane ports provide access for remote 
manipulators and diagnostics, while 32 angled ports provide access to the divertor regions for utilities and diagnostics. FIRE is being designed mechanically to accommodate 3,000 full field, full power pulses and 30,000 pulses at $2 / 3$ field. The repetition time at full field and full pulse length will be $<3 \mathrm{hr}$, with shorter times at reduced parameters. The fusion energy production of $5 \mathrm{TJ}$ (similar to BPX) produces a lifetime neutron dose to the TF insulating material at the inboard midplane of $\approx 1.5 \times 10^{10}$ Rads which is consistent with the polyimide insulation being considered.

The power densities on the divertor plates are $\sim 5 \mathrm{MWm}^{-2}$ for detached operation and $\sim 25$ $\mathrm{MWm}^{-2}$ for attached operation. The divertor plasma-facing components are tungsten "brush" targets mounted on copper backing plates, similar to a concept developed by the ITER R\&D activity. The outer divertor plate is water-cooled, while the baffle and inner divertor targets are inertially cooled. The first wall is comprised of Be plasma-sprayed onto copper tiles which do not need active cooling for pulses $<15 \mathrm{~s}$. The large neutron wall loading $\left(3 \mathrm{MWm}^{-2}\right)$ at fusion power of $200 \mathrm{MW}$ contributes significantly to the first wall and vacuum vessel heating. Either a modest reduction in fusion power due to lower H-mode threshold assumptions, or improved cooling will be required for a $20 \mathrm{~s}$ pulse length. The plasma facing materials were chosen to reduce the tritium inventory in the first wall. Sixteen cryopumps closely coupled to the divertor chambers, but behind sufficient neutron shielding - provide pumping $\left(\geq 100 \mathrm{~Pa} \mathrm{~m}^{3} / \mathrm{s}\right)$ for D-T and He ash during the pulse. Pellet injection scenarios using direct injection inside the magnetic axis and guided inside launch will be incorporated, and are expected to provide a modest increase in fusion reactivity due to density profile peaking while minimizing tritium consumption. The in-device tritium inventory will be determined primarily by the cycle time of the divertor cryopumps, and can range from $<2 \mathrm{~g}$ for regeneration overnight to $\sim 20 \mathrm{~g}$ for monthly regeneration.

The possibility of using only high conductivity (OFHC) copper in the TF coil is being investigated. This lower strength material would require the addition of TF coil bucking on the central solenoid coils near the midplane. Initial results suggest that $11.5 \mathrm{~T}$ could be produced with a flattop of $\approx 40 \mathrm{~s}$ using about $1 / 2$ of the electrical power that the baseline $\mathrm{BeCu} \mathrm{TF}$ coil design requires. The limitation on burn time for both $\mathrm{BeCu}$ and OFHC designs is the power handling capability of plasma facing components and the vacuum vessel.

A number of important physics issues remaining to be addressed during the design phase, and then resolved during the experimental program. These include generic issues such as: mitigation and avoidance of disruptions and vertical displacement events, H-mode power threshold, effects of neoclassical tearing modes, detached divertor operation with good confinement, and divertor/edge plasma modeling under high power conditions.

FIRE, coupled with a non-burning steady-state superconducting advanced tokamak in an international multi-machine strategy, would address many of the objectives identified for Next Physics Steps in Magnetic Fusion (Fig. 1). The FIRE design study is a U. S. national activity managed through the Virtual Laboratory for Technology and is supported by DOE. PPPL work supported by DOE Contract \# DE-AC02-76CHO3073. 
The Princeton Plasma Physics Laboratory is operated by Princeton University under contract with the U.S. Department of Energy.

\author{
Information Services \\ Princeton Plasma Physics Laboratory \\ P.O. Box 451 \\ Princeton, NJ 08543
}

Phone: 609-243-2750

Fax: 609-243-2751

e-mail: pppl_info@pppl.gov

Internet Address: http://www.pppl.gov 